\section{Composite Mould} Based on 3D PrintingCOMBO3D

Hybrid manufacturing and newly designed materials open new possibilities in production technology.

\section{Dr Swen Zaremba}

Technical University of Munich, Germany

\section{Combining the strengths of production processes}

The component manufactured out of the tool is a flap using the resin transfer moulding process. Essentially a casting process in which liquid resin is injected into a carbon fibre textile in a close mould under pressure. With high surface requirents for acedynamic surfaces for components like this are challenging to design and manufacture as it is, but to design and manufacture as
the project went beyond this.

Using large-scale AM using highperformance polymers, the mould shells were 3D printed and finished by milling in a hybrid manufacturing approach. This allows the mould to be functionalised with internal features to accelerate the
cycle time and make the process more robust. Curved injection line guides give new freedom in positioning injection points, ensuring complete mould fillins and surface conformal fluid channels introduce heat where it's needed
shortening the production cycle. As AM produces a workpiece near its final shape, only a few millimetres had to be milled off the surfaces to ensure the required smooth finish. This reduces the mould production time to a few weeks rather than months and minimises material waste in the process, making otherwise expensive materials feasible.

\section{Materials designed for AM}

3D printing with polymers is increasingly difficult with higher material performance, but some applications require highperforming materials.

One of the highest performing polymer groups is the semi-crystalline polyaryletherketone (PAEK) group with members such as PEEK and PEKK found in aerospace parts, medical implants, and even seals and pipes in oil extraction. These materials are difficult to print on small machines with sophisticated heated build chabs. Printing a three-metre-long mould in a sing a three metre long mould in would be impossible if not for the newly designed materials for $3 \mathrm{D}$ printing.
NewPAEKgrades withslowcrystallisation speeds offer the best of both worlds in polymers. During processing, the polymers stay amorphous for a long time making them easy to process with high bonding strength between the layers and low warping. Yet, they develop the excellent properties desirable for parts upon crystallisation, like a longer lifespan of the mould thanks to the improved chemical and thermal stability. With these materials, an otherwise impossible process is not only feasible but stable enough to succeed on the first attempt in the project.

Changing the way we design Finding the right material and getting it to work with your machine is not all that is necessary to make a part 3D printable. AM has design constrain that need to be considered just as established production processes have. What the draft angle or undercuts are to casting, or tool accessibility is to milling the maximum overhang and minimum feature size are to $3 D$ printing. Instead of creating a drilled hole by designing a block of material and then removing a cylinder from it, when designing a hole for AM, its space is left empty, and the materia is added around it. Hybrid manufacturing using $\mathrm{AM}$ to create a workpiece for milling that will become a mould for casting requires high flexibility in design thinking. Drilled holes are always round and straight and can be precise enough for positioning pins. Printed holes can

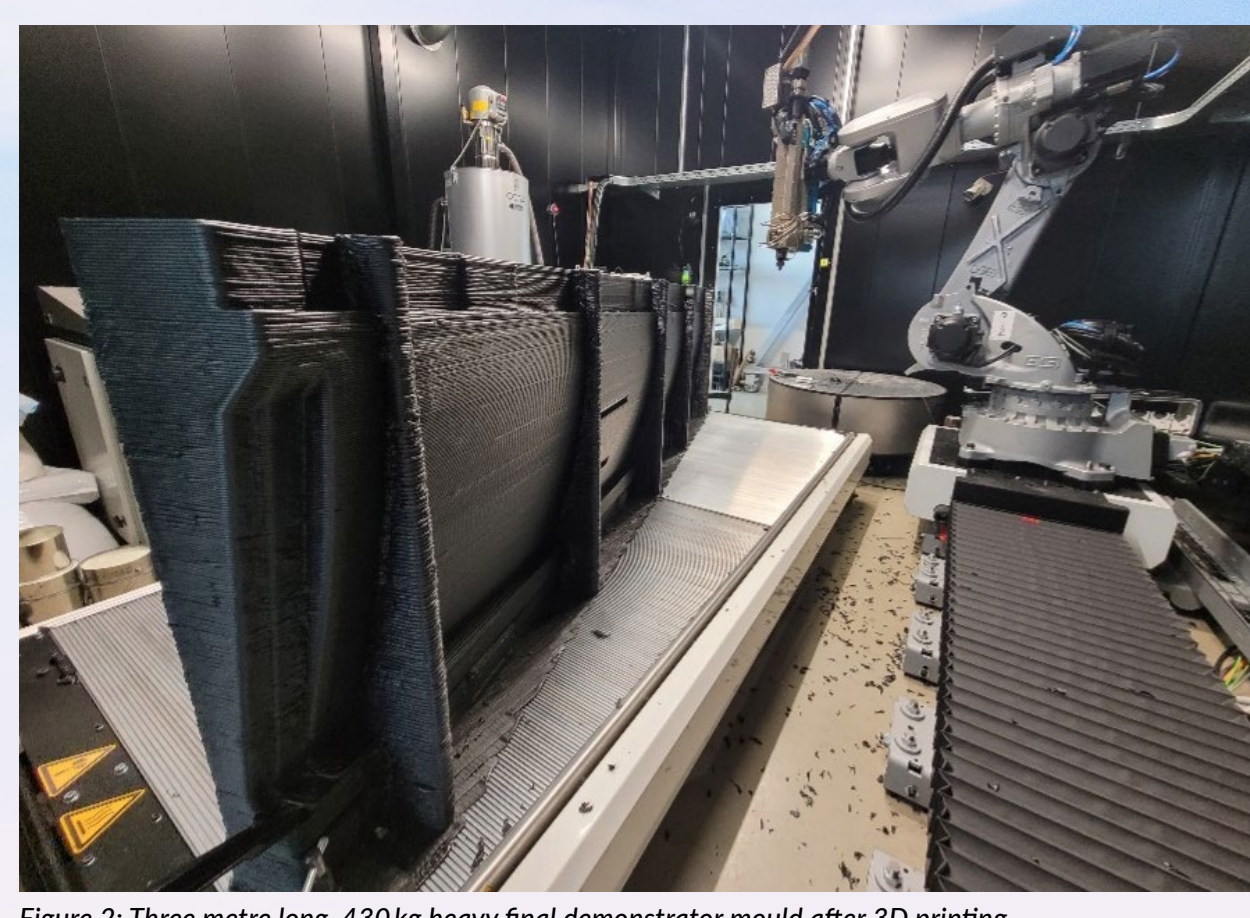
Figure 2: Three metre long $430 \mathrm{~kg}$ heavy final demonstrator mould after $3 \mathrm{D}$ printing.

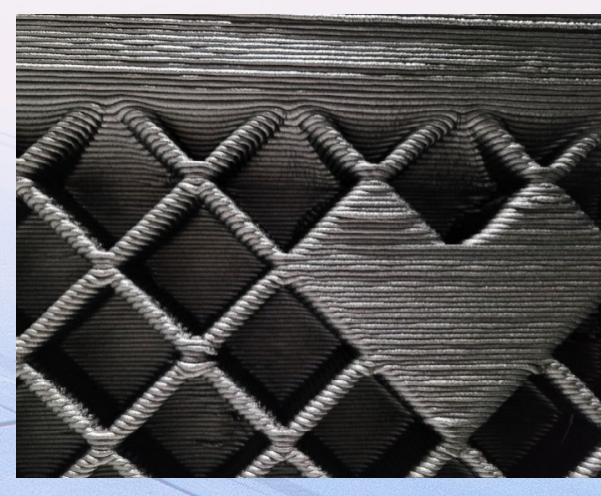

and more functional parts:

- The resin inlets can be positioned where they are needed in casting, not where they are possible to machine by guiding the injection lines around temperature inhomogeneity and flow resistance.

Figure 3: Overhac
lines in AM parts.

Mould cavities have smooth surfaces es but come with the precision of milling.

following a curved surface or turning - Standard pellet feedstock with fast around a bend. If done correctly, hybrid procurement, but single piece mould manufacturing combines the strengths of shells without gaps between them both technologies in synergy for better and minimised assembly times.

The Clean Sky 2 project COMBO3D investigated using additive

manufacturing (AM) to accelerate the cycle, development and procurement lead time of aerospace tooling. 


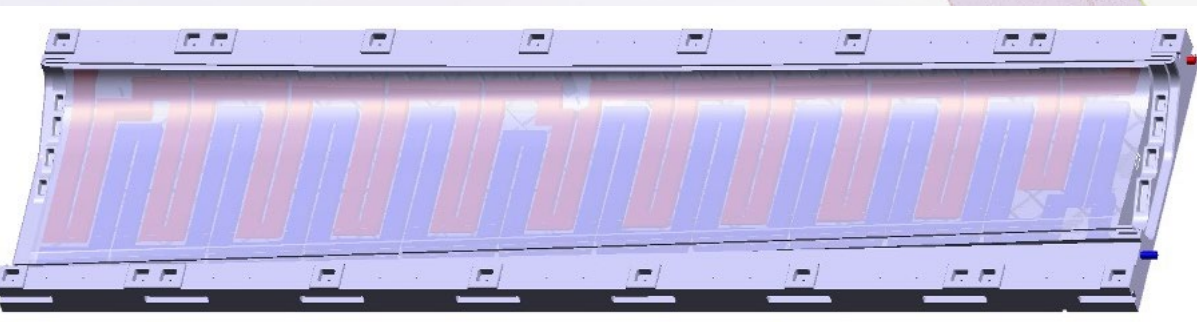

Figure 4: Rendering of mould shell with visualised flow channels.

Tooling, jigs and fixtures paving the way for AM adoption in industry

Designing an AM part as you would any other and viewing $A M$ as an isolate process will never lead to an optimal solution. However, embracing the
benefits of AM, considering its constraints and integrating it into a production chain has the potential to revolutionise how we make things. But before we see $3 \mathrm{D}$ printed aircraft or other vehicles, there a lot left to learn about AM.

Tooling, jigs and fixtures are involved in almost every production process across industries. They are often very complex sometimes one of a kind and, in most cases, the final customer will never know about them. This makes them ideal candidates to replace and gain experience in using AM without the risk f reputation loss, which would result in technology tiredness.

Starting in the protected environment of their own workshop, the risks of potential failure are manageable. Exposing a printed part to a production environment with solvents, high process temperatures and possibly high forces can be very challenging compared to already established products. If successful, AM can reduce the costs of existing processes and make them more competitive while at the same time gaining experience with the process and improving its reliability, repeatability and predictability.

The next development steps following the project will be the integration of continuous fibres to reduce the CTE of the mould to match the parts, potentially replacing nickel alloys like Invar in tooling, and the direct additive manufacture of secondary structural aircraft parts.
PROJECT SUMMARY

COMBO3D aimed to produce a mould for a passenger aircraft flaperon using 3D printing and high-performance polymers. Taking
advantage of new design freedom, the advantage of new design freedom, the mould was designed to be functionalised for a faster and more robust production
of the aerospace composite part.

\section{PROJECT LEAD}

Patrick Consul currently conducts research in large-scale additive manufacturing at the Chair of Carbon Composites of the Technical University of Munich, investigating the process-hiduced material anisotropy. He leads the research topte for mach he desibe and automation, focusing on automated

\section{PROJECT PARTNERS}

This project would not have been possible without the contributions of the project partners Victrex Ltd from the UK in material development, ALPEX Technologies in tooling design and machining, LeichtmetallKompetenzzentrum Ranshofen LKR in fluid channel optimisation and the Technical University of Munich large-scale AM, thermal simulation and design for additive manufacturing. The project topic was mat under the Clean Sky 2 joint undertaking.

CONTACT DETAILS

Dr Swen Zaremba

Chair of Carbon Composites, Technical University of Munich, Boltzmannstr. 15,

85748 Garching, Germany

Iㅍ +49(0)8928915092

凶info.lcc@ed.tum.de

(1) https://www.asg.ed.tum.de/en/ https://www.asg.ed.tum.de/en/

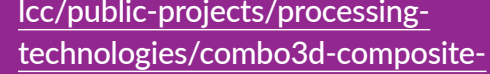
mould-too/

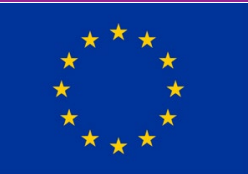

FUNDING

This project has received funding from the European
Union's Horizon 2020 research and innovation Union's Horizon 2020 research and innovation
programme under grant agreement No. 831851.

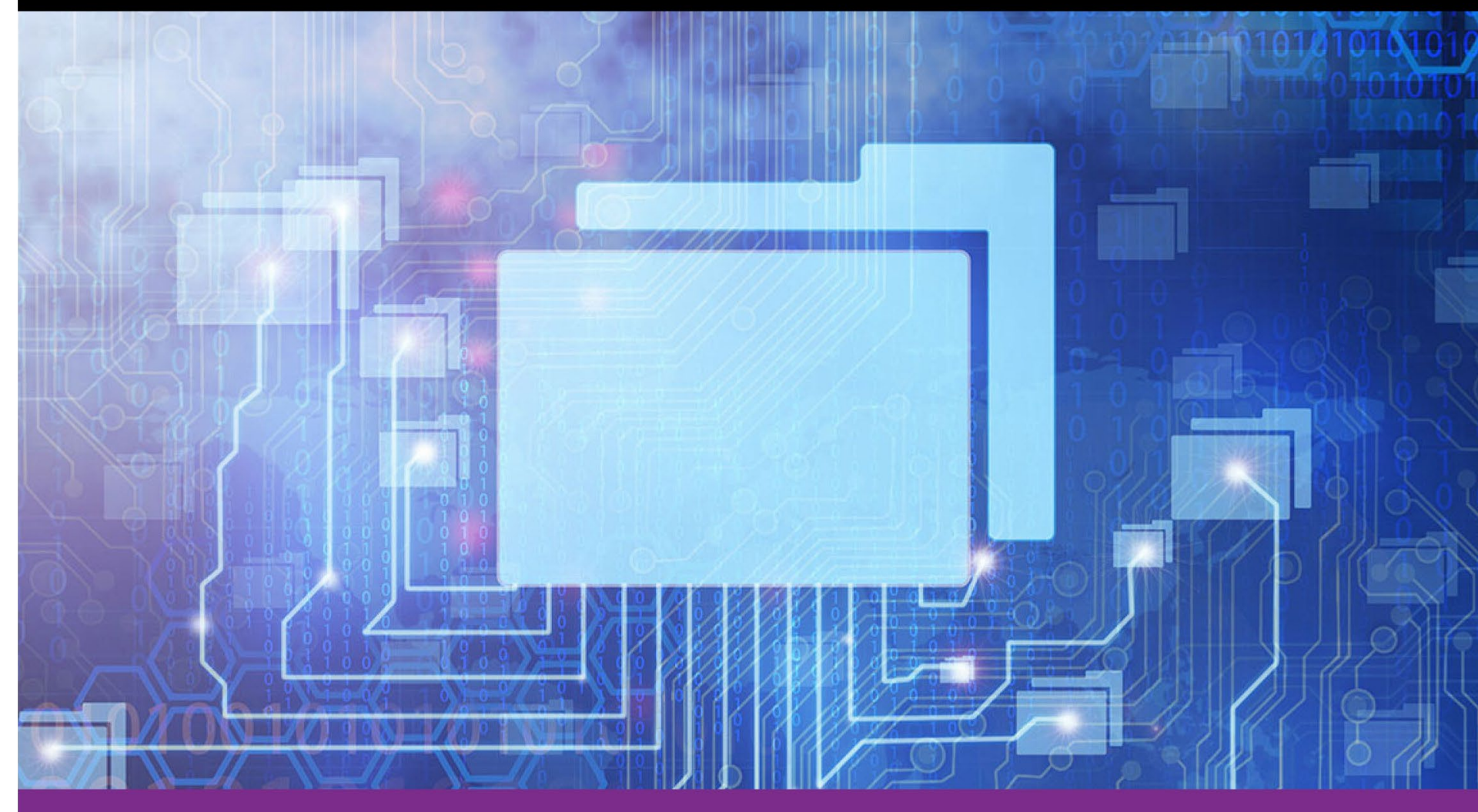

\section{PROJECT REPOSITORY}

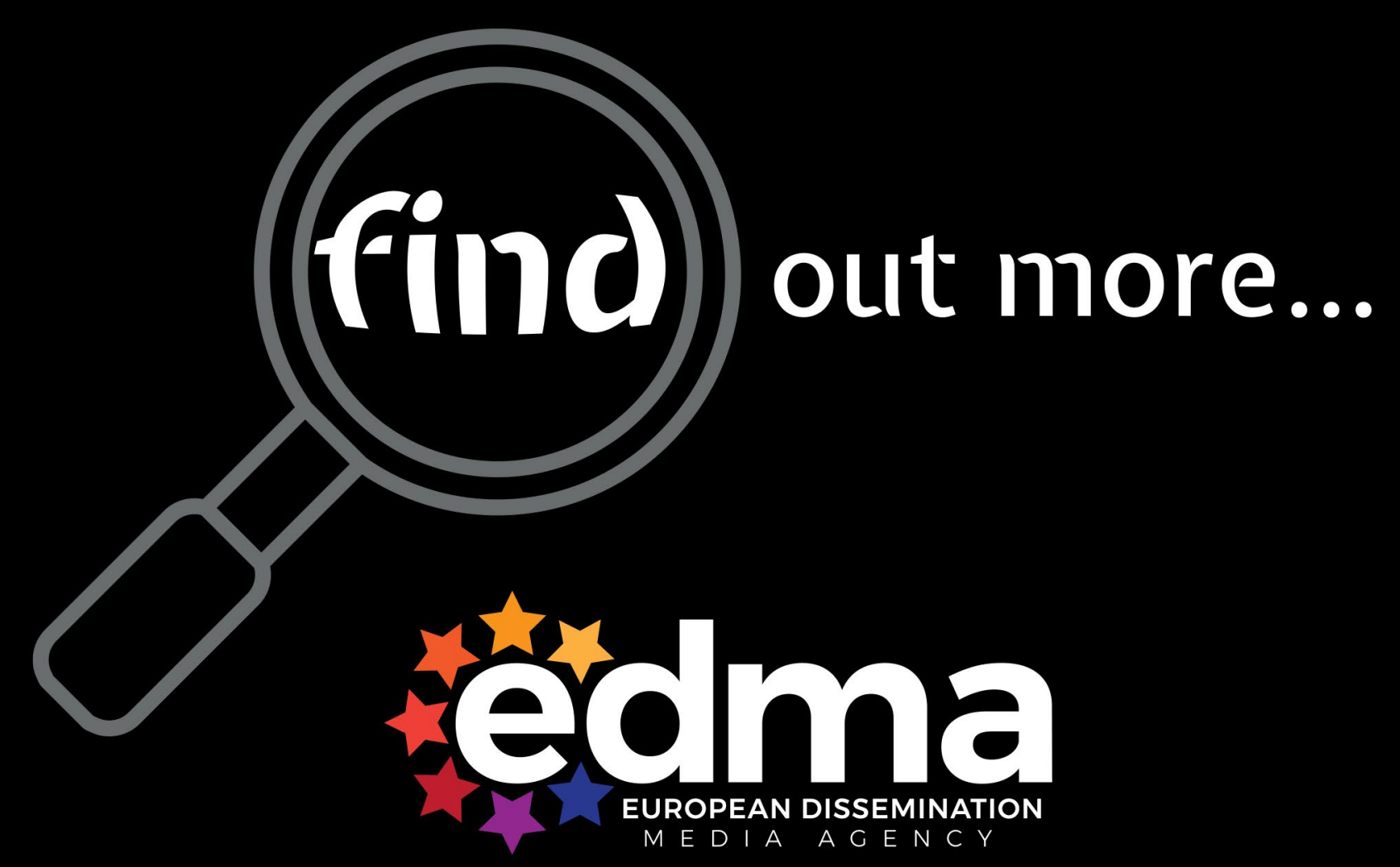

Disseminate - Communicate - Educate 